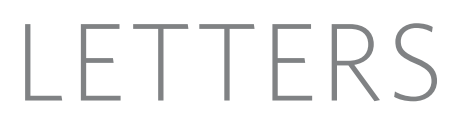

\title{
Functional diversification of closely related ARF-GEFs in protein secretion and recycling
}

\author{
Sandra Richter ${ }^{1}$, Niko Geldner ${ }^{1} \dagger$, Jarmo Schrader ${ }^{1}$, Hanno Wolters ${ }^{1}$, York-Dieter Stierhof ${ }^{2}$, Gabino Rios ${ }^{3} \dagger$, \\ Csaba Koncz ${ }^{3}$, David G. Robinson ${ }^{4} \&$ Gerd Jürgens $^{1}$
}

Guanine-nucleotide exchange factors on ADP-ribosylation factor GTPases (ARF-GEFs) regulate vesicle formation in time and space by activating ARF substrates on distinct donor membranes ${ }^{1}$. Mammalian GBF1 (ref. 2) and yeast Gea1/2 (ref. 3) ARF-GEFs act at Golgi membranes, regulating COPI-coated vesicle formation. In contrast, their Arabidopsis thaliana homologue GNOM (GN) is required for endosomal recycling, playing an important part in development ${ }^{4}$. This difference indicates an evolutionary divergence of trafficking pathways between animals and plants, and raised the question of how endoplasmic reticulum-Golgi transport is regulated in plants. Here we demonstrate that the closest homologue of GNOM in Arabidopsis, GNOM-LIKE1 (GNL1; NM_123312; At5g39500), performs this ancestral function. GNL1 localizes to and acts primarily at Golgi stacks, regulating COPI-coated vesicle formation. Surprisingly, GNOM can functionally substitute for GNL1, but not vice versa. Our results suggest that large ARF-GEFs of the GBF1 class perform a conserved role in endoplasmic reticulum-Golgi trafficking and secretion, which is done by GNL1 and GNOM in Arabidopsis, whereas GNOM has evolved to perform an additional plant-specific function of recycling from endosomes to the plasma membrane. Duplication and diversification of ARF-GEFs in plants contrasts with the evolution of entirely new classes of ARF-GEFs ${ }^{5}$ for endosomal trafficking in animals, which illustrates the independent evolution of complex endosomal pathways in the two kingdoms.

Large ARF-GEFs are represented by two subfamilies conserved among eukaryotes: the GBF1 clade, including mammalian GBF1, yeast Gea1/2 and Arabidopsis GNOM; and the BIG clade, including mammalian BIG1/2, yeast Sec7 and Arabidopsis BIG1-5 (refs 5, 6) (Fig. 1a). Whereas GBF1 and Gea1/2 have been localized to the $\mathrm{Golgi}^{2,3,7}$, and a double knockout for Gea1/2 is cell-lethal ${ }^{3,7}$, GNOM performs a plant-specific endosomal recycling function important for development but not essential for cell viability ${ }^{4,8,9}$. Large ARFGEFs are the molecular targets for brefeldin A (BFA), which causes reversible inhibition of vesicle trafficking ${ }^{4}$. BFA traps sensitive ARF-GEFs on the membrane by blocking the guanine-nucleotide exchange reaction ${ }^{10}$. BFA sensitivity or resistance can be engineered by site-directed mutagenesis, without affecting protein function ${ }^{11}$, providing a unique tool to selectively inhibit specific ARF-GEFs to probe their function in vivo. This approach revealed that GNOM is involved in endosomal recycling of PIN1, a polarly localized component of auxin efflux carriers ${ }^{4}$. We now extend this approach to identify the ARF-GEF responsible for endoplasmic reticulum (ER)-Golgi trafficking. BFA was shown to cause the release of COPI coats from membranes in mammalian cells ${ }^{12,13}$. In Arabidopsis, however, BFA treatment has no effect on Golgi structure
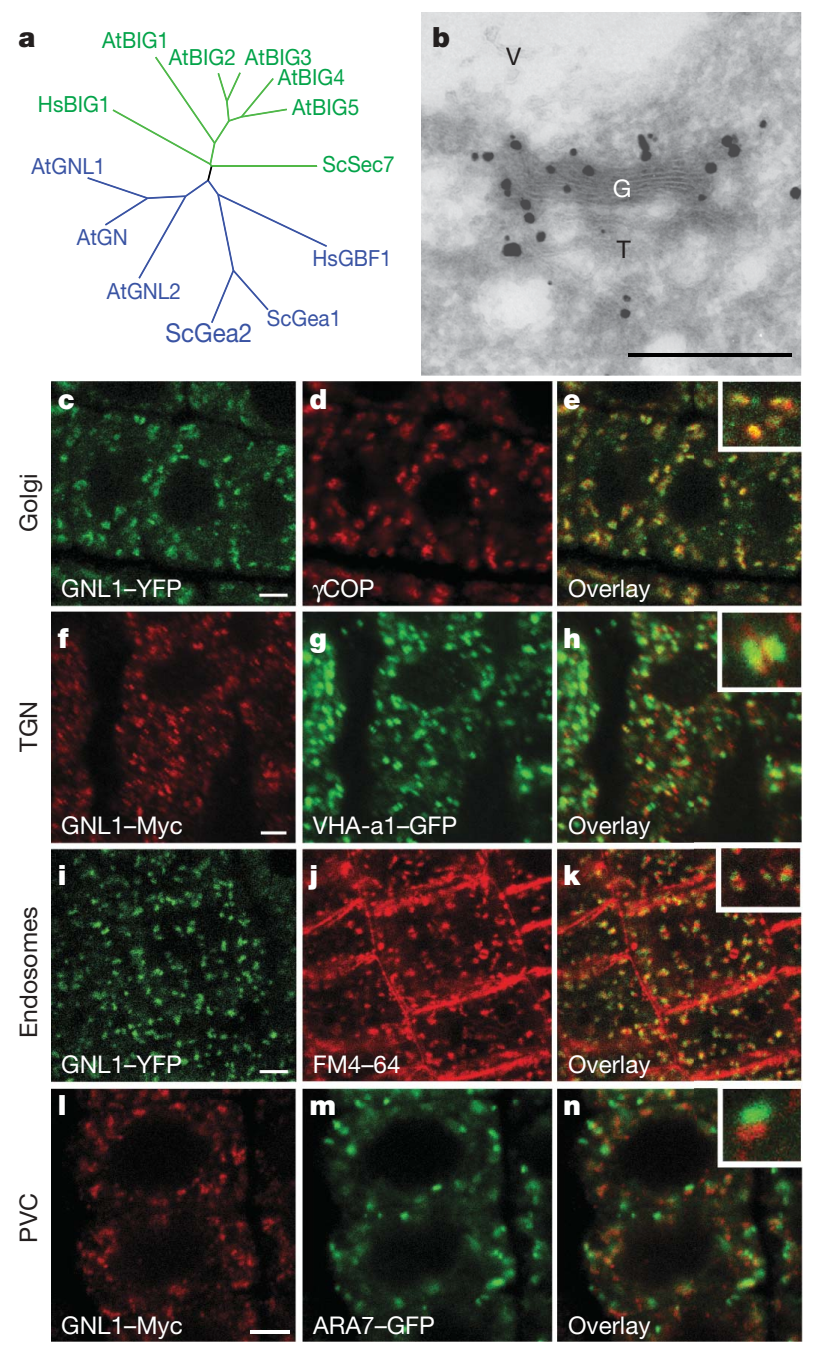

Figure $\mathbf{1}$ | Identification of the GNL1 compartment. a, Unrooted phylogenetic tree of large ARF-GEFs from Arabidopsis (At), human (Hs) and yeast $(\mathrm{Sc})$. b, Immunogold localization of GNL1-YFP on an ultrathin cryosection. G, Golgi; T, TGN; V, vacuole. Scale bar, $500 \mathrm{~nm}$. c-n, Immunofluorescence localization of GNL1 and endomembrane markers. Scale bars, $4 \mu \mathrm{m}$. c-e, GNL1-YFP partially colocalizes with Golgi marker $\gamma$ COP. No colocalization was observed for GNL1-Myc and TGN marker VHA-a 1-GFP (f-h), GNL1-YFP and endocytic tracer FM4-64 (i-k) or GNL1-Myc and prevacuolar compartment marker ARA7-GFP (I-n). 
or membrane association of the COPI subunit $\gamma \mathrm{COP}^{4,14}$, suggesting that a BFA-resistant ARF-GEF mediates this process. Evident candidates are the two closest homologues of GNOM. We decided to focus on ubiquitously expressed GNOM-LIKE1 (GNL1) because GNL2 seems to be pollen-specific ${ }^{15}$ (Supplementary Fig. 2).

To identify the endomembrane compartment at which GNL1 acts, we generated plants expressing functional GNL1-Myc or GNL1-YFP (yellow fluorescent protein). GNL1 was localized relative to compartment-specific markers (Fig. 1; Supplementary Table 1). GNL1 partially co-localized with the Golgi marker $\gamma \mathrm{COP}^{16}$ but did not, or only marginally so, with the trans-Golgi network (TGN) marker VHA-a1$\mathrm{GFP}^{17}$, which was consistent with the immunogold-localization of GNL1 (Fig. 1b-h; Supplementary Fig. 3f). In contrast, GNL1 did not co-localize with ARA7-GFP labelling endosomes/pre-vacuolar compartments ${ }^{18,19}$ nor the endocytic tracer FM4-64 (ref. 4) (Fig. 1i-n). These results suggest a role for GNL1 in Golgi-related trafficking.

BFA treatment of Arabidopsis seedlings causes aggregation of endosomal membrane vesicles (so-called BFA compartments), to which GNOM has been localized ${ }^{4,14}$. Predominantly at the periphery of BFA compartments, GNL1 partially co-localized with the Golgi markers $\gamma$ COP and N-ST-YFP ${ }^{20}$, and also overlapped with VHA-a1GFP and FM4-64 (Fig. 2a-d; Supplementary Fig. 4). To conditionally inactivate GNL1 by BFA treatment, we introduced an engineered BFA-sensitive GNL1 into a gnl1 knockout background. This switch to BFA-sensitivity of GNL1 caused a dramatic change in BFA effects. We now observed a release of $\gamma \mathrm{COP}$ into the cytosol and a block in ER-Golgi traffic, as highlighted by the accumulation of the Golgi marker N-ST-YFP in the ER as well as the fusion of Golgi stacks with the ER (Figs 2g, h and 3c, d; Supplementary Fig. 3). Consistently, BFA-sensitive GNL1 localized to the ER but also co-localized with FM4-64 in BFA compartments (Fig. 2f; Supplementary Fig. 5; see Supplementary Fig. $3 \mathrm{~g}$ and $\mathrm{h}$ for immunogold localization of GNL1-YFP). This indicates that GNL1 activity is required for the integrity of Golgi stacks and for COPI-coated vesicle formation. To analyse whether GNL1 might also have another role in trafficking, we studied the membrane association of another major vesicle coat protein, clathrin (Supplementary Fig. 6). In contrast to $\gamma \mathrm{COP}$, however, clathrin was not released into the cytosol by BFA treatment of BFA-sensitive GNL1, which is consistent with the localization of GNL1 and clathrin to adjacent but distinct compartments (Supplementary Fig. 6a-c, g-i). As a consequence of blocking anterograde traffic of newly synthesized proteins from the ER to the Golgi, the TGN marker VHA-a1-GFP also accumulated at the ER (Fig. 2i). Thus, GNL1 is a major regulator of ER-Golgi trafficking and protein secretion.

If GNL1 has such an important role in membrane traffic one would expect inactivation of the GNL1 gene to be lethal. We identified a T-DNA insertion knockout line for GNL1 (Supplementary Fig. 7a). Surprisingly, the gnll mutant plants were viable and fertilealthough short and bushy-and pollen competitiveness was reduced to $76 \%$ (Fig. 3i). Another strong allele, gnl1-2, isolated as a secretion mutant, showed a very similar phenotype (ref. 21; our unpublished

Figure 2 | Localization of BFA-resistant and BFA-sensitive GNL1 after BFA treatment. BFA-resistant (a-d) or BFA-sensitive ( $\mathbf{f}-\mathbf{i})$ GNL1 in gnl1 mutant background localized relative to compartment markers after BFA-treatment. a, f, Endocytic tracer FM4-64 (red) in BFA compartments colocalizes partially with GNL1-YFP and is surrounded by GNL1-YFP aggregates (a), whereas BFA-sensitive GNL1-YFP localizes to ER (arrow) and colocalizes with FM4-64 in small BFA compartments (f, asterisk). b, g, Golgi marker $\gamma$ COP (red) partially overlaps with GNL1-YFP (b) but is released into the cytosol when GNL1-YFP is BFA-sensitive (g). c, $\mathbf{h}$, Golgi marker N-ST-YFP (green) colocalizes partially with GNL1-Myc (c) but relocates to the ER when GNL1-Myc is BFA-sensitive (h). $\mathbf{d}, \mathbf{i}$, TGN marker VHAa1-GFP (green) accumulates in BFA compartments (d) but is trapped in the ER when GNL1-Myc is BFA-sensitive (i). e, $\mathbf{j}$, Golgi localization of $\gamma \mathrm{COP}$ (e, red) and N-ST-YFP (j, green) restored by BFA-resistant GN in BFAsensitive GNL1, gnl1 mutant background. All scale bars, $4 \mu \mathrm{m}$. observations). These results indicate that GNL1 is not essential. Consistently, gnl1 mutant cells displayed slightly abnormal Golgi stacks with cisternae laterally expanded by approximately 35\% (Fig. 3a, b; Supplementary Fig. 3i). However, BFA treatment of gnll mutant cells caused fusion of the Golgi with the ER, suggesting
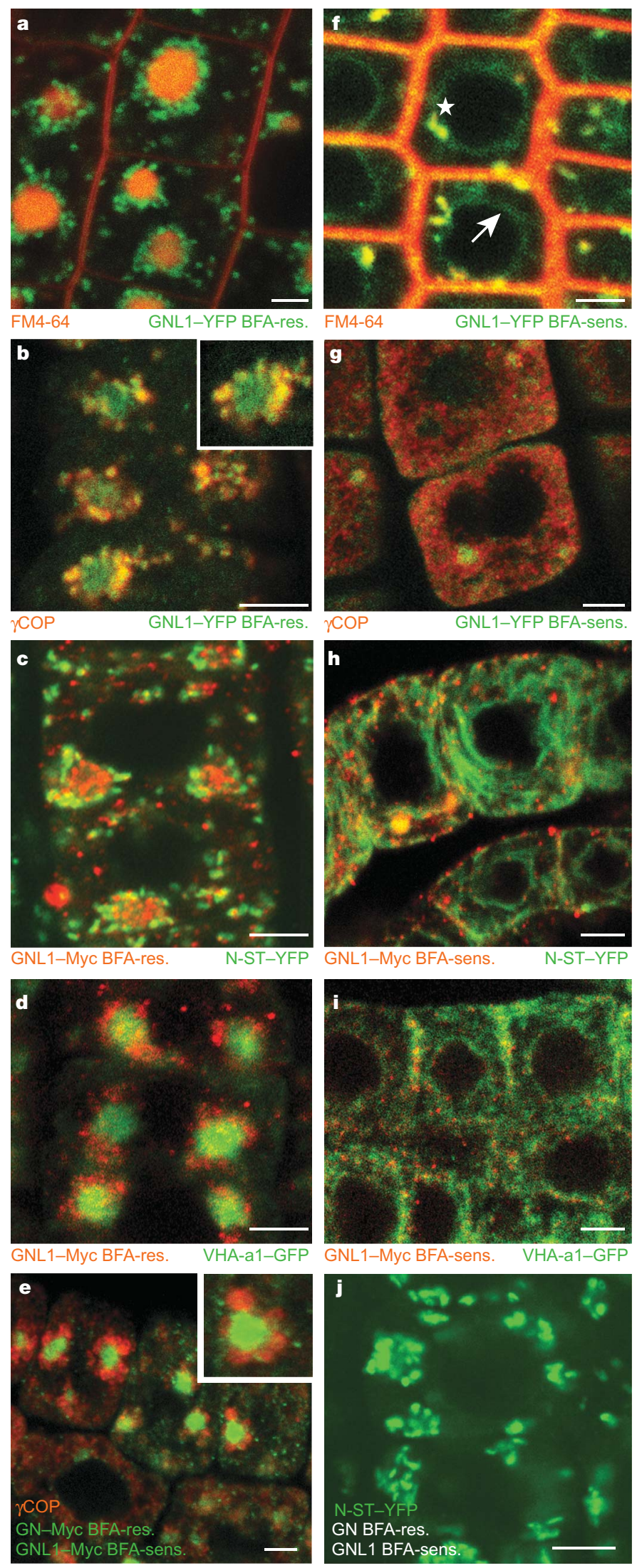
that another, BFA-sensitive ARF-GEF is also involved in ER-Golgi trafficking (Fig. 3c, d; Supplementary Fig. 3e). One candidate would be GNOM, although GNOM is involved in endosomal recycling ${ }^{4}$. In addition, gnom mutant embryo cells did not display major Golgi abnormalities (Supplementary Fig. 3c) and GNOM did not colocalize with GNL1 (Fig. 3e). However, when both ARF-GEFs were
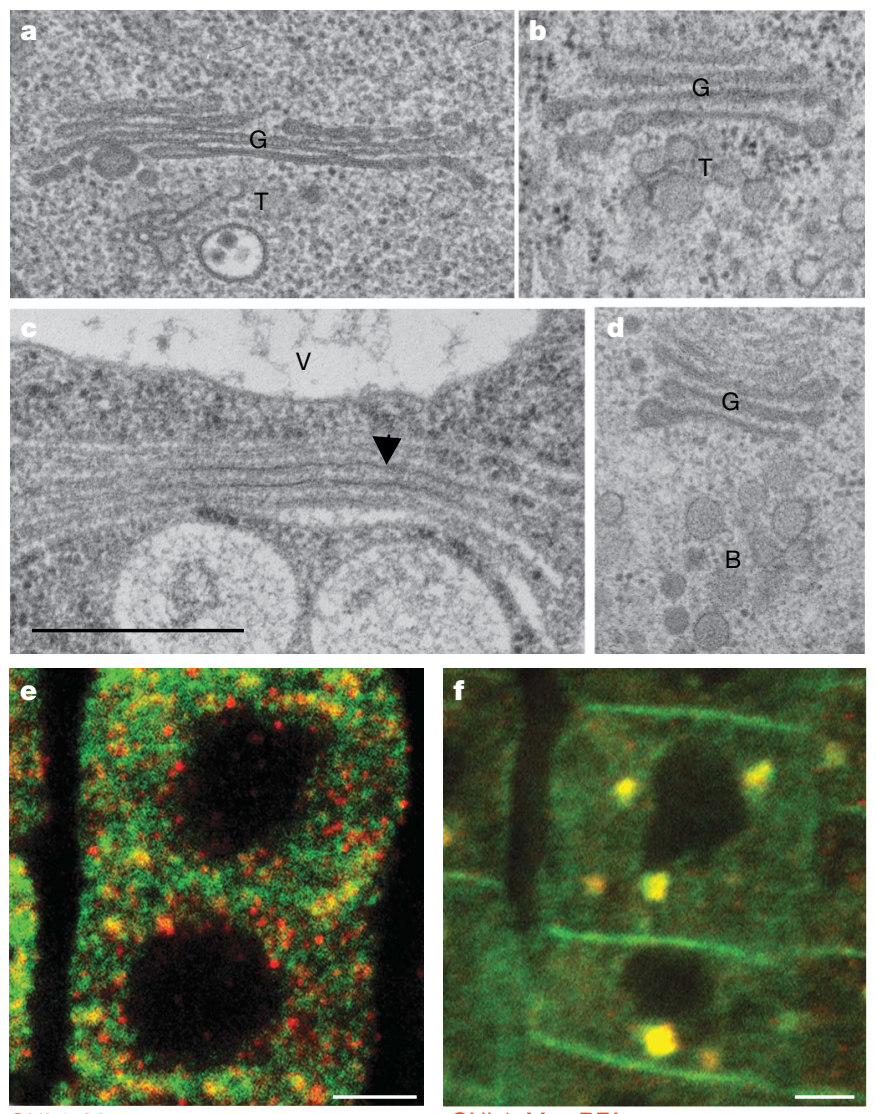

GNL1-Myc

GNL1-Myc BFA sens.

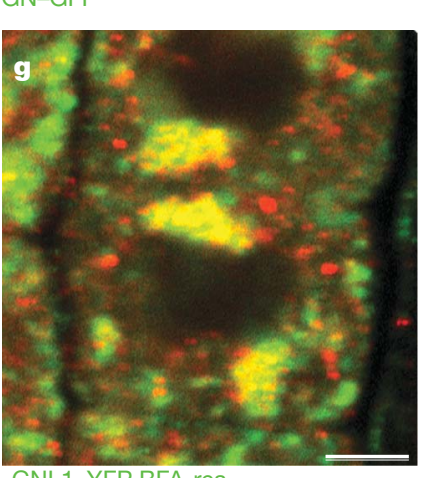

GNL1-YFP BFA-res.

GN-Myc BFA-res.
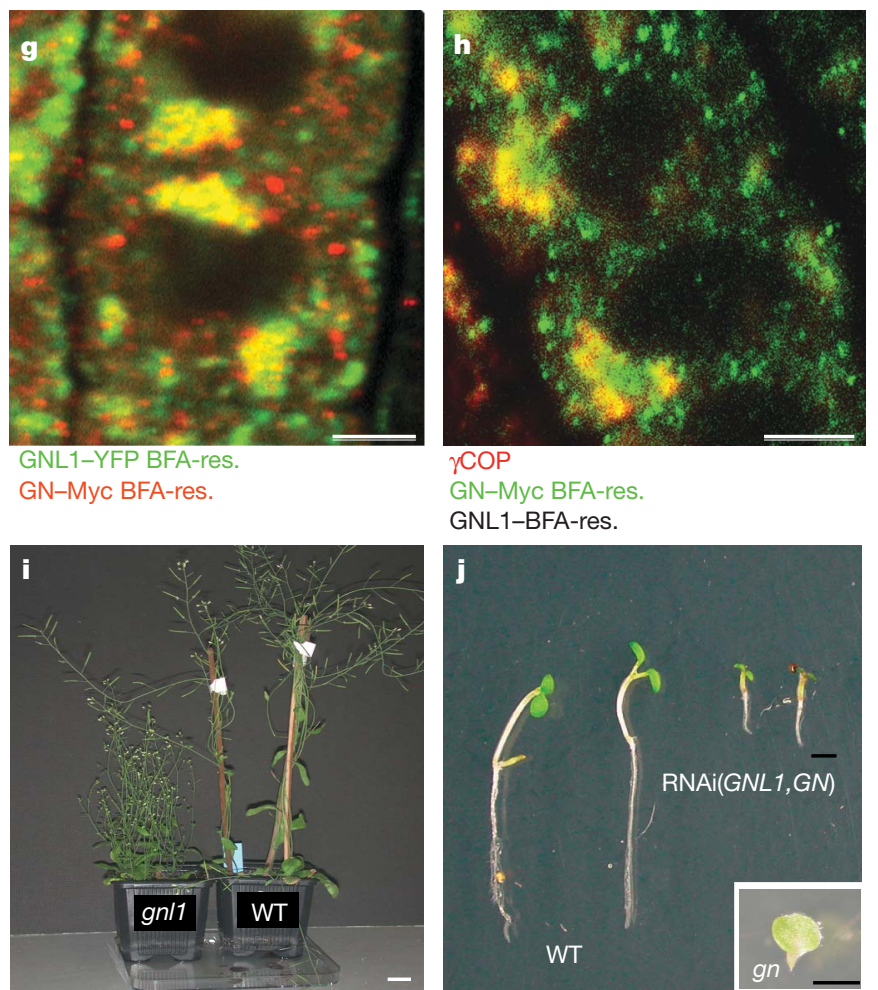

YCOP

GN-Myc BFA-res.

GNL1-BFA-res.

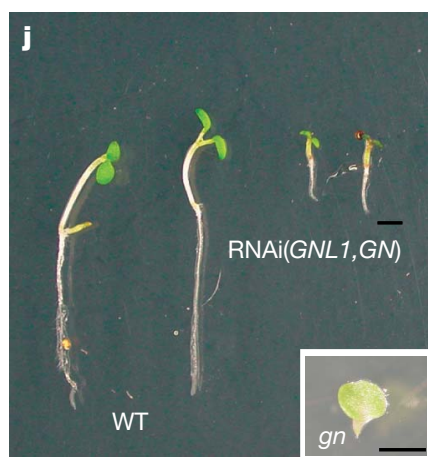

rendered either BFA-resistant or BFA-sensitive they co-localized partially or completely, respectively, after BFA treatment (Fig. 3f, g). In addition, the BFA-resistant form of both ARF-GEFs also co-localized partially with $\gamma \mathrm{COP}$, suggesting the additional presence of GNOM protein at Golgi stacks (Figs $3 \mathrm{~h}$ and $2 \mathrm{~b}$ ). To determine whether GNOM can substitute for GNL1 function, we analysed the localization of $\gamma$ COP and N-ST-YFP in BFA-treated BFA-sensitive GNL1, gnl1 mutant plants expressing engineered BFA-resistant GNOM (Fig. 2e, j). Surprisingly, the wild-type localization of the two markers was restored, although the BFA sensitivity of the two ARF-GEFs was reversed, indicating that BFA-resistant GNOM could compensate for the inactivation of BFA-sensitive GNL1 (Fig. 2e, j, compare with Fig. $2 \mathrm{~b}, \mathrm{~g}$ and $2 \mathrm{c}$, h, respectively). Thus, although GNOM might play only a minor part in ER-Golgi trafficking, it can take over the Golgi function of GNL1.

We also tested whether GNL1 can replace GNOM in the PIN1 endosomal recycling required for polar localization of PIN1 at the plasma membrane ${ }^{4}$ (Fig. $4 \mathrm{a}-\mathrm{d}$ ). Polar targeting of PIN1 to the plasma membrane was only observed in BFA-treated seedlings when GNOM was BFA-resistant, regardless of BFA resistance or sensitivity of GNL1 (Fig. 4b, d). Thus, GNL1 has no obvious role in endosomal recycling. Interestingly, the BFA compartments were very small when both GNL1 and GNOM were BFA-sensitive, but attained their normal size when one or the other ARF-GEF was BFA-resistant. This suggests that BFA compartments are formed from both GNL1-dependent secretory and endocytic membrane material, although we cannot rule out a minor contribution of GNL1 to PIN1 recycling (compare Fig. 4c with $4 \mathrm{a}, \mathrm{d})$.

The capacity of GNOM to replace GNL1 cellular function could account for the observed, weak gnll mutant phenotypes (Fig. 3i). Indeed, gnll gnom double mutants lacking both ARF-GEF activities were gametophytic lethal (Supplementary Table 2). This indicates that both ARF-GEFs are functionally redundant and that their double knockout affects some fundamental cellular function that leads to lethality even before fertilisation. Interestingly, other allele combinations with reduced ARF-GEF activity were also lethal, suggesting that the combined level of activity is critical (Supplementary Table 2). To determine their requirements in diploid somatic cells, both GNL1 and GNOM were simultaneously downregulated by RNA interference (RNAi) expression (Supplementary Fig. 7b, c). The RNAi(GNL1, GN) expressing seedlings eventually died after 10-12 days of growth (Fig. 3j). Their ultrastructural analysis revealed abnormalities of the Golgi stacks, supporting our notion that ERGolgi trafficking requires ARF-GEF function provided by both GNL1 and GNOM (Supplementary Fig. 3b).

To examine whether there are additional non-overlapping functions of GNL1 and GNOM, we performed promoter swaps and analysed the chimaeric genes for their ability to replace the two mutated genes. Surprisingly, GNL1-GNOM was able to rescue both gnom and gnl1 mutants, whereas GNOM-GNL1 only rescued gnl1 but not gnom mutants (Fig. 4e; Supplementary Fig. 8a-c). Thus, GNOM and GNL1

Figure 3 | Phenotype of gnl1 mutants and relationship of GNL1 to GN. a-d, Golgi morphology of gnll mutant $(\mathbf{a}, \mathbf{c})$ in comparison to wild-type (b, d) after BFA treatment (c, d). a, b, Golgi of gnl1 mutant (a) laterally expanded compared to wild-type in untreated controls. c, d, After $50 \mu \mathrm{M}$ BFA for $1 \mathrm{~h}$, Golgi fuses with ER (arrowhead) in gnl1 mutant (c), whereas Golgi morphology is retained in wild type (d). B, BFA compartment; G, Golgi; T, TGN; V, vacuole. Scale bar for a-d, $500 \mathrm{~nm}$. e-g, Subcellular localization of GNL1 and GN. e, No colocalization of GNL1-Myc (red) with GN-GFP (green) in untreated cells. Nearly complete colocalization of BFAsensitive versions in gnll mutant (f) and partial colocalization of BFAresistant versions $(\mathbf{g})$ after BFA treatment. $\mathbf{h}$, Partial colocalization of BFAresistant GN with Golgi marker $\gamma$ COP. Scale bars for $\mathbf{e}-\mathbf{h}, 4 \mu \mathrm{m}$.

i, j, Phenotype of gnll mutant plants and RNAi(GNL1, GN) seedlings. i, gnl1 mutant plant ( $g$ nll; left), wild type (WT; right). Scale bar, $2 \mathrm{~cm}$. j, Seedling phenotypes of RPS5A-RNAi(GNL1, GN) (right) compared with wild type (left), 6 days after germination. Inset, $g n$ mutant. Scale bar, $2 \mathrm{~mm}$. 

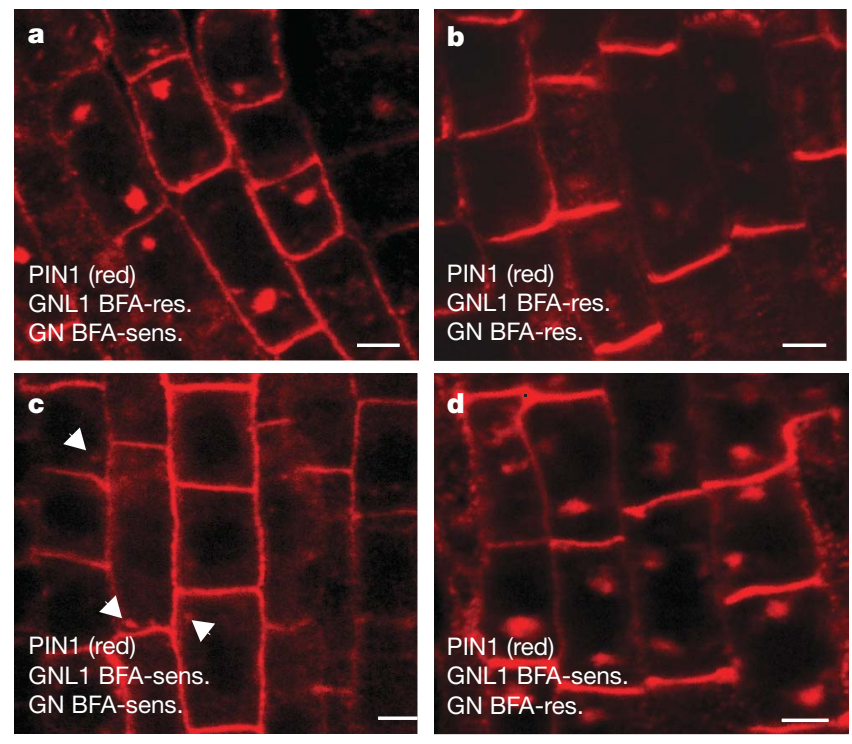

e

\begin{tabular}{|c|c|c|}
\hline Mutant & Transgene & GN-GNL1-Myc \\
\hline gn/gn & Rescue & No rescue \\
\hline gn/1/gn/1 & Rescue & Rescue \\
\hline
\end{tabular}

f
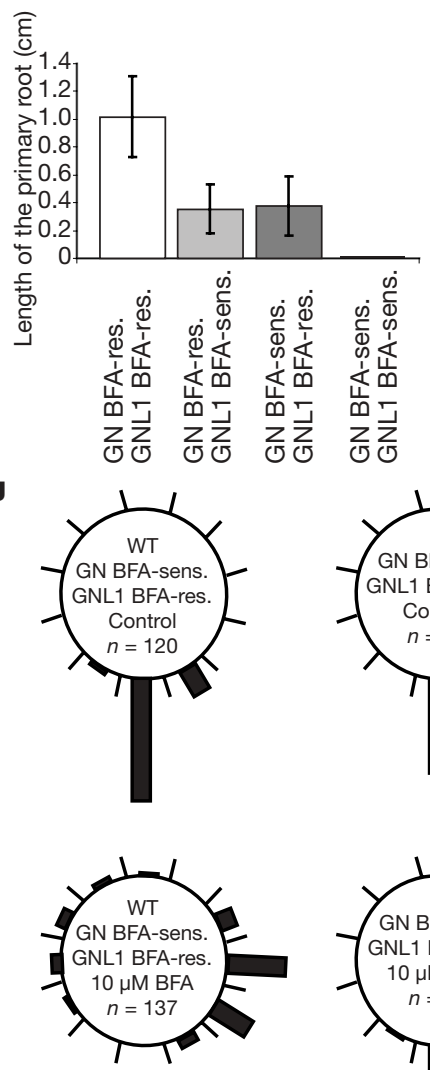

ह

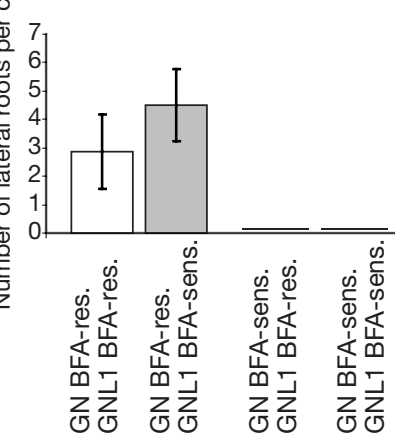

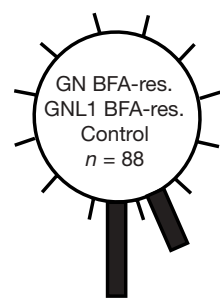

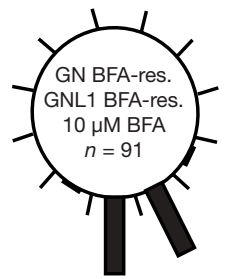

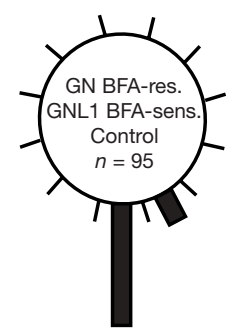

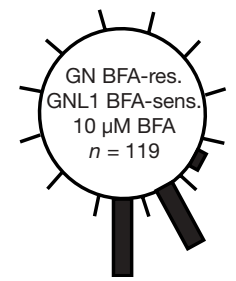

share one common, essential function in ER-Golgi trafficking, whereas only GNOM has another, unique essential function in endosomal recycling.

We then used BFA to determine the relative contributions of GNL1 and GNOM in different BFA-sensitive developmental processes such as seed germination, primary root growth, lateral root
Figure 4 | Functional relationship between GN and GNL1. a-d, PIN1

localization after BFA treatment. a, GN BFA-sensitive, GNL1 BFA-resistant (wild type): PIN1 is localized in BFA compartments and symmetrically at the plasma membrane. $\mathbf{b}, \mathbf{d}$, Polar localization of PIN1 at the plasma membrane and variable accumulation of PIN1 in BFA compartments when only GN (b) or both GN and GNL1 (d) are BFA-resistant. c, GN and GNL1 BFAsensitive in gnl1 mutant background: PIN1 is localized in very small BFA compartments (arrowheads) and symmetrically at the plasma membrane (similar to wild type, Fig. 4a). Scale bars for a-d, $4 \mu \mathrm{m}$. e, Promotor swaps between GN and GNL1 suggest asymmetric functional redundancy. f, g, Seedlings expressing different combinations of BFA-resistant (res.) versus BFA-sensitive (sens.) GNL1 and GNOM (GN) in gnll mutant background (except for wild-type) were treated with BFA. f, Left panel, additive effects of GNL1 and GN in primary root growth. Right panel, lateral root formation is mainly dependent on GN but not on GNL1. Error bars indicate standard deviation; $n=46$ (GN BFA-res., GNL1 BFA-res.), 46 (GN BFA-res., GNL1 BFA-sens.), 22 (GN BFA-sens., GNL1 BFA-res., which corresponds to wild type), 25 (GN BFA-sens., GNL1 BFA-sens.). g, Root gravitropism is a GN-dependent process (upper panel, control; lower panel, BFA treatment).

initiation and root gravitropism. The two ARF-GEFs acted interchangeably in seed germination (Supplementary Fig. 9). Unexpectedly, expression of either BFA-resistant ARF-GEF, GNL1 or GNOM, was sufficient to sustain root growth at half the rate of roots expressing both BFA-resistant ARF-GEFs, indicating that primary root growth requires both GNL1 and GNOM function (Fig. 4f, left). In contrast, both lateral root initiation and root gravitropism were dependent on BFA-resistant GNOM, whereas BFA sensitivity or resistance of GNL1 had no effect of its own (Fig. 4f, right; 4g). This requirement for GNOM-specific ARF-GEF function is related to its role in endosomal recycling of PIN1 (ref. 4, 22).

Our results offer a surprising explanation for why ARF-GEFs of the GBF1 clade act in different trafficking pathways in plants than in mammals or yeast. There are two closely related ARF-GEFs, GNL1 and GNOM, that share the ancestral Golgi-associated function of GBF1-type ARF-GEFs in Arabidopsis and probably in other flowering plants as well (Supplementary Fig. 1). This was not noticed before because, unlike in mammals, there was no BFA effect on the localization of $\gamma \mathrm{COP}$ in wild-type Arabidopsis, although GNOM is BFA-sensitive $e^{4,14}$. In contrast, a rapid effect of BFA on the Golgi morphology and the localization of $\gamma \mathrm{COP}$ was described for tobacco BY-2 cells ${ }^{23}$, suggesting that the tobacco GNL1 orthologue is BFAsensitive. Indeed, we found a BFA-sensitive signature for the rice putative orthologue of GNL1 (Supplementary Fig. 10). Our data imply that the Golgi-localized ARF-GEF function is ancestral and conserved among eukaryotes, although the plant lineage has apparently undergone gene duplication such that two closely related ARFGEFs perform the GBF1-equivalent function. GNOM acquired an additional specific function in endosomal recycling of plasma membrane proteins that is not shared by GNL1. Unlike plants, animals have evolved new classes of small or medium-sized ARF-GEFs named cytohesin, EFA and BRAG, which are involved in endosomal trafficking. In addition, a member of the BIG class of large ARF-GEFs named BIG2 acts not only at the TGN, like its close relative BIG1, but also at recycling endosomes ${ }^{24}$. Thus, the elaboration of post-Golgi trafficking seems to have evolved independently in the two major multicellular lineages, whereas the regulation by ARF-GEF of ERGolgi traffic was already in place in unicellular eukaryotes.

\section{METHODS SUMMARY}

Molecular biology. For expression of tagged GNL1, a complementing genomic fragment was transformed into gnll mutants, and BFA sensitivity was engineered by replacing methionine with leucine in the SEC7 domain. For RNAi(GN, GNL1) analysis, fragments of GNL1 and GN coding sequence in tandem in sense and antisense direction were introduced into a two-component vector for expression by RPS5A-GAL4 activator ${ }^{25}$. Transcript levels were analysed by RTPCR. Promoter swaps involved 2-kb promoter fragments and were introduced into gnll and emb30-1 (ref. 8) heterozygous plants. 
Expression analysis. Protein blots $^{26}$, immunofluorescence localization ${ }^{4,26,27}$, FM4-64 staining ${ }^{4}$, BFA treatment ${ }^{4}$, immunolocalization on cryosections $s^{28}$, electron microscopy $\mathrm{y}^{28}$ and physiological tests ${ }^{4}$ were performed as described.

Bioinformatics. An unrooted phylogenetic tree of SEC7 domains from different ARF-GEFs was derived from sequence alignment using ClustalW (www.ebi.ac. uk/clustalw).

Full Methods and any associated references are available in the online version of the paper at www.nature.com/nature.

\section{Received 18 February; accepted 30 May 2007.}

1. Shin, H. W. \& Nakayama, K. Guanine nucleotide-exchange factors for arf GTPases: their diverse functions in membrane traffic. J. Biochem. 136, 761-767 (2004).

2. Zhao, X. et al. GBF1, a cis-Golgi and VTCs-localized ARF-GEF, is implicated in ERto-Golgi protein traffic. J. Cell Sci. 119, 3743-3753 (2006).

3. Peyroche, A., Courbeyrette, R., Rambourg, A. \& Jackson, C. L. The ARF exchange factors Gea1p and Gea2p regulate Golgi structure and function in yeast. J. Cell Sci. 114, 2241-2253 (2001).

4. Geldner, N. et al. The Arabidopsis GNOM ARF-GEF mediates endosomal recycling, auxin transport, and auxin-dependent plant growth. Cell 112, 219-230 (2003).

5. Cox, R., Mason-Gamer, R. J., Jackson, C. L. \& Segev, N. Phylogenetic analysis of Sec7-domain-containing Arf nucleotide exchangers. Mol. Biol. Cell 15, 1487-1505 (2004).

6. Mouratou, B. et al. The domain architecture of large guanine nucleotide exchange factors for the small GTP-binding protein Arf. BMC Genom. 6, 20 (2005).

7. Spang, A., Herrmann, J. M., Hamamoto, S. \& Schekman, R. The ADP ribosylation factor-nucleotide exchange factors Gea1p and Gea2p have overlapping, but not redundant functions in retrograde transport from the Golgi to the endoplasmic reticulum. Mol. Biol. Cell 12, 1035-1045 (2001).

8. Mayer, U., Büttner, G. \& Jürgens, G. Apical-basal pattern formation in the Arabidopsis embryo: studies on the role of the gnom gene. Development 117, 149-162 (1993).

9. Steinmann, T. et al. Coordinated polar localization of auxin efflux carrier PIN1 by GNOM ARF GEF. Science 286, 316-318 (1999).

10. Cherfils, J. \& Melancon, P. On the action of Brefeldin A on Sec7-stimulated membrane-recruitment and GDP/GTP exchange of Arf proteins. Biochem. Soc. Trans. 33, 635-638 (2005).

11. Peyroche, A. et al. Brefeldin A acts to stabilize an abortive ARF-GDP-Sec7 domain protein complex: involvement of specific residues of the Sec7 domain. Mol. Cell 3, 275-285 (1999).

12. Barzilay, E., Ben-Califa, N., Hirschberg, K. \& Neumann, D. Uncoupling of brefeldin A-mediated coatomer protein complex-I dissociation from Golgi redistribution. Traffic 6, 794-802 (2005).

13. Niu, T. K., Pfeifer, A. C., Lippincott-Schwartz, J. \& Jackson, C. L. Dynamics of GBF1, a Brefeldin A-sensitive Arf1 exchange factor at the Golgi. Mol. Biol. Cell 16, 1213-1222 (2005).

14. Geldner, N., Friml, J., Stierhof, Y.-D., Jürgens, G. \& Palme, K. Auxin transport inhibitors block PIN1 cycling and vesicle trafficking. Nature 413, 425-428 (2001).

15. Schmid, M. et al. A gene expression map of Arabidopsis thaliana development. Nature Genet. 37, 501-506 (2005).

16. Pimpl, P. et al. In situ localization and in vitro induction of plant COPI-coated vesicles. Plant Cell 12, 2219-2236 (2000).

17. Dettmer, J., Hong-Hermesdorf, A., Stierhof, Y.-D. \& Schumacher, K. Vacuolar H $\mathrm{H}^{+}$ ATPase activity is required for endocytic and secretory trafficking in Arabidopsis. Plant Cell 18, 715-730 (2006)

18. Ueda, T., Uemura, T., Sato, M. H. \& Nakano, A. Functional differentiation of endosomes in Arabidopsis cells. Plant J. 40, 783-789 (2004).

19. Lee, G. J., Sohn, E. J., Lee, M. H. \& Hwang, I. The Arabidopsis rab5 homologs Rha1 and Ara7 localize to the prevacuolar compartment. Plant Cell Physiol. 45, $1211-1220$ (2004).
20. Grebe, M. et al. Arabidopsis sterol endocytosis involves actin-mediated trafficking via ARA6-positive early endosomes. Curr. Biol. 13, 1378-1387 (2003).

21. Teh, O. \& Moore, I. An ARF-GEF acting at the Golgi and in selective endocytosis in polarized plant cells. Nature doi:10.1038/nature06023 (this issue).

22. Geldner, N. et al. Partial loss-of-function alleles reveal a role for GNOM in auxin transport-related, post-embryonic development of Arabidopsis. Development 131, 389-400 (2004).

23. Ritzenthaler, C. et al. Reevaluation of the effects of brefeldin A on plant cells using tobacco Bright Yellow 2 cells expressing Golgi-targeted green fluorescent protein and COPI antisera. Plant Cell 14, 237-261 (2002).

24. Shin, H. W., Morinaga, N., Noda, M. \& Nakayama, K. BIG2, a guanine nucleotide exchange factor for ADP-ribosylation factors: its localization to recycling endosomes and implication in the endosome integrity. Mol. Biol. Cell 15, 5283-5294 (2004).

25. Weijers, D., Van Hamburg, J. P., Van Rijn, E., Hooykaas, P. J. \& Offringa, R. Diphtheria toxin-mediated cell ablation reveals interregional communication during Arabidopsis seed development. Plant Physiol. 133, 1882-1892 (2003).

26. Lauber, M. H. et al. The Arabidopsis KNOLLE protein is a cytokinesis-specific syntaxin. J. Cell Biol. 139, 1485-1493 (1997).

27. Müller, A. et al. AtPIN2 defines a locus of Arabidopsis for root gravitropism control. EMBO J. 17, 6903-6911 (1998).

28. Völker, A., Stierhof, Y.-D. \& Jürgens, G. Cell cycle-independent expression of the Arabidopsis cytokinesis-specific syntaxin KNOLLE results in mistargeting to the plasma membrane and is not sufficient for cytokinesis. J. Cell Sci. 114, 3001-3012 (2001).

29. Rios, G. et al. Rapid identification of Arabidopsis insertion mutants by nonradioactive detection of T-DNA tagged genes. Plant J. 32, 243-253 (2002).

30. Vieten, A. et al. Functional redundancy of PIN proteins is accompanied by auxindependent cross-regulation of PIN expression. Development 132, 4521-4531 (2005).

31. Song, J., Lee, M. H., Lee, G. J., Yoo, C. M. \& Hwang, I. Arabidopsis EPSIN1 plays an important role in vacuolar trafficking of soluble cargo proteins in plant cells via interactions with clathrin, AP-1, VTI11, and VSR1. Plant Cell 18, 2258-2274 (2006).

Supplementary Information is linked to the online version of the paper at www.nature.com/nature.

Acknowledgements We thank N. Takada and L. Müller for technical assistance, A. Vieten, J. Friml, F. El-Kasmi, G. Strompen and K. Steinborn for screening the Cologne T-DNA insertion lines, K. Schumacher, I. Hwang, M. Grebe, A. Schlereth and D.Weijers for providing materials, O. Teh and I. Moore for sharing unpublished material and results, and N. Anders, U. Mayer, K. Schumacher and D. Weigel for critically reading the manuscript and suggestions. We especially thank N. Anders for advice and discussions. This work was supported by an EMBO long-term Fellowship to J.S. and by grants from the Human Frontier in Science Program Organization and the SFB 446 of the Deutsche Forschungsgemeinschaft to G.J.

Author Contributions S.R. carried out most of the experiments, N.G. initiated the project, J.S. generated the RNAi and promoter-swap lines, H.W. generated the ARA7-GFP marker line, Y.-D.S. performed the electron microscopy analysis and immunogold localization experiments, G.R. and C.K. provided the T-DNA collection, D.G.R. generated antisera against markers, and G.J. and S.R. designed the experiments, discussed the results and wrote the manuscript.

Author Information Reprints and permissions information is available at www.nature.com/reprints. The authors declare no competing financial interests. Correspondence and requests for materials should be addressed to G.J. (gerd.juergens@zmbp.uni-tuebingen.de). 


\section{METHODS}

Plant material and growth conditions. Arabidopsis wild-type (ecotype Col-0) and mutant lines carrying gnom alleles R5 (ref. 22) or emb30-1 (ref. 8) have been described. Plant growth conditions were as described ${ }^{22}$. Seedlings (Col-0 ecotype) were grown from surface-sterilized seeds on vertical agar plates at $24^{\circ} \mathrm{C}$ for 5 days under long day conditions ${ }^{22}$.

gnl1 mutant. The gnl1-1 T-DNA insertion line (ecotype Col-0) was isolated as described $^{29}$. The T-DNA insertion is located in the first exon at 102 base pairs downstream of the ATG and confers hygromycin resistance. The plants were selected on plates containing $15 \mu \mathrm{g} \mathrm{ml}^{-1}$ hygromycin (Gibco). The genotype was characterized by two PCRs: one to detect the T-DNA insertion (sense primer, $5^{\prime}$ GATTGAGCCAAGAAGTTGGGGCGAG-3'; antisense primer, 5' -CTGGGAATGGCGAAATCAAGGCAT- $3^{\prime}$ ), another to amplify the wild-type gene sequence spanning the T-DNA insertion site (sense primer, $5^{\prime}$-ACCAAAAGGGTAGAGTTGAAAAGGG-3'; antisense primer: 5'-TACATTTCTCCTCATCACAGCCAAA-3').

Generating transgenic plants. An 8.8-kb genomic GNL1 fragment containing 2-kb of 5' UTR and 1-kb of 3' UTR from BAC clone MUL8 complemented the gnll mutant phenotype. Primer-extension PCR was used to insert $3 \times$ Myc or YFP tag at the C-terminus as well as to introduce the leucine 696 (TTG) to methionine 696 (ATG) mutation in the SEC7 domain. All constructs were transformed into gnl1/GNL1 heterozygous plants, and at least five gnl1 mutant lines each were established for BFA-resistant and BFA-sensitive GNL1 transgenes and shown to complement the bushy plant phenotype of gnll mutants.

For RNAi(GN, GNL1) analysis, 172 bp of GNL1 and 162 bp of GNOM coding sequence were cloned in tandem in sense and antisense orientation, introduced into the UAS vector of the GAL4》UAS two-component expression system and transformed into Col- 0 plants. $\mathrm{T}_{2}$ plants were crossed with RPS5A-GAL4 plants ${ }^{25}$, and the $\mathrm{F}_{1}$ seedling progeny with abnormal phenotypes were analysed.

A 2-kb promotor fragment of GNL1 and a 2.1-kb promoter fragment of GN were fused to genomic fragments for promoter swapping. These constructs were introduced into gnl1/GNL1 as well as emb30-1/GN heterozygous plants ${ }^{8}$.

All $\mathrm{T}_{1}$ plants were selected by spraying seedlings on soil with BASTA $(1: 1,000$; AgrEvo) or by adding kanamycin $\left(50 \mu \mathrm{g} \mathrm{ml}^{-1}\right.$; Sigma) to plates.

Immunofluorescence localization. Five-day-old seedlings were incubated in $1 \mathrm{ml}$ of liquid growth medium $(\times 0.5 \mathrm{MS}$ medium, $1 \%$ sucrose, $\mathrm{pH} 5.8)$ containing $50 \mu \mathrm{M}$ BFA (Invitrogen, Molecular Probes) for $1 \mathrm{~h}$ (or, $100 \mu \mathrm{M}$ for $2 \mathrm{~h}$ in double labelling for GN and GNL1) at room temperature in 24-well cell-culture plates. Incubation was stopped by fixation with $4 \%$ paraformaldehyde in MTSB. Immunofluorescence staining was performed as described ${ }^{26}$ or with an InsituPro machine (Intavis) ${ }^{27}$.

Antibodies used were: mouse anti-c-Myc mAb 9E10 (Santa Cruz Biotechnologies) diluted 1:600; mouse anti-GFP (Roche) 1:600; rabbit antiGFP (Invitrogen, Molecular Probes) 1:600; rabbit anti-PIN1 (ref. 30) 1:1,000; rabbit anti-At $\gamma$ COP (ref. 16) 1:1,000; and anti-clathrin ${ }^{31} 1: 500$. FITC or Cy3conjugated secondary antibodies (Dianova) were diluted 1:600.
Epitope-tagged or GFP-fusion markers were transformed or crossed into tagged GNL1 lines: RPS5A-GFP-ARA7 (ref. 18) (GFP-ARA7; cloned into pGrIIK RPS5a-tNOS; ref. 25), VHA-a1-GFP ${ }^{17}, \mathrm{~N}-\mathrm{ST}-\mathrm{YFP}^{20}, \mathrm{GN}-3 \times \mathrm{Myc}^{4}$ (BFA-sensitive and resistant) and BFA-sensitive GN-GFP .

Fluorescence signals of GFP- or YFP-tagged proteins were enhanced by staining with anti-GFP antibody in fixed cells. Live cell imaging was performed with $2 \mu$ M FM4-64 (Invitrogen, Molecular Probes).

Acquisition and processing of fluorescence images. Fluorescence images were acquired at $512 \times 512$ pixels with the confocal laser scanning microscope TCSSP2 from Leica, using the $\times 63$ water-immersion objective and Leica software. All images were processed with Adobe Photoshop CS2 only for adjustment of contrast and brightness.

Immunolocalization on cryosections, and electron microscopy. Ultrastructural analysis of high-pressure frozen, freeze-substituted and resin-embedded root tips and embryos, and immunogold labeling using silver-enhanced Nanogold (Nanoprobes, USA) of ultrathin cryosections were carried out as described $^{17}$.

Physiological tests. To investigate primary root growth and lateral root formation, 5-day-old seedlings were transferred to plates with $20 \mu \mathrm{M}$ BFA and analysed after 7 additional days.

For measurement of gravitropic responses, 4-day-old seedlings were transferred to plates containing $10 \mu \mathrm{M}$ BFA. After 1 day, the plates were turned by $135^{\circ}$. Pictures were taken $12 \mathrm{~h}$ later and deviation from the gravity vector was measured.

For the germination assay, surface-sterilized seeds were plated on agar plates $(\times 0.5 \mathrm{MS}$ medium, $1 \%$ sucrose, $\mathrm{pH} 5.8)$ containing $20 \mu \mathrm{M}$ BFA. After two days of stratification, the plates were incubated at $23^{\circ} \mathrm{C}$ for 4 days before seed germination rates were determined.

Analysis of transcript levels in gnl1 mutants and RNAi(GN, GNL1) lines. RNA isolation was performed using Plant RNAeasy Kit from Qiagen. RT-PCR was done with Revert Aid H Minus First Strand cDNA Synthesis Kit (Fermentas). PCR on gnl1 cDNA from plants was performed with sense primer $5^{\prime}$ CTGGGAATGGCGAAATCAAGGCAT-3' and antisense primer 5'-CTTTTTTCTCCAGAATTCGG-3'. PCR on cDNA from RNAi seedlings was done with sense primer $5^{\prime}$-GTGCAGTTTTGGCTGTGATG-3' and antisense primer $5^{\prime}$ CTTTTTTCTCCAGAATTCGG-3' to investigate GNL1 transcript level. For GN, sense primer $5^{\prime}$-TACACTTGTCAACAGAGCTGGTAGC- $3^{\prime}$ and antisense primer 5' -TCTGTCATTATATGCAAATCATATGGAGAAGCCG-3' were used. Western blot analysis. Five-day-old seedlings were used for protein extraction ${ }^{26}$. Protein extracts were loaded onto a $12 \%$ polyacrylamide gel and transferred onto a PVDF membrane. Rabbit anti-GNOM(SEC7) antiserum diluted 1:2,000 and anti-rabbit horseradish peroxidase (POD)-conjugated secondary antibody (Roche) 1: 5,000 were used to detect endogenous GNOM protein. Detection was performed with BM chemiluminescence blotting substrate (Roche).

Phylogenetic tree. SEC7 domain sequences of ARF-GEFs from different species, corresponding to the GN SEC7 domain, were aligned by ClustalW (www.ebi.ac.uk/clustalw) and the phylogenetic tree was drawn with TreeViewer. 